\title{
A Comprehensive Methodology for Opportunistic Spectrum Access based on Residual White Space Distribution
}

\author{
Manuj Sharma and Anirudha Sahoo \\ Dept. of Computer Science and Engineering \\ Indian Institute of Technology Bombay \\ Mumbai, India \\ manuj@it.iitb.ac.in, sahoo@it.iitb.ac.in
}

\begin{abstract}
Assuming that Secondary Users (SU) have channel occupancy data (channel idle and busy durations) of Primary Users (PU), this paper presents a methodology to fit primary channel occupancy data to a distribution. Then we show how SUs can opportunistically transmit in the white spaces based on residual white space distribution while keeping probability of interference (with the PUs) below a predefined threshold. We also present analytical formulation for the average throughput of the secondary user and validate it through simulation. Our simulation results show that using the proposed methodology, SU's transmission always remains below the interference threshold and the channel efficiency increases with minimal impact on PU application performance for low values of interference threshold.
\end{abstract}

Categories and Subject Descriptors:C.2.5 [Local and Wide-Area Networks]: Access schemes

General Terms: Theory, Performance

Keywords: Opportunistic spectrum access, residual white space, cognitive radio

\section{INTRODUCTION}

Opportunistic Spectrum Access (OSA) enables Secondary Users (SU) to use those parts of the spectrum band that are not currently utilized in space or time by any Primary User (PU). The SU identifies the idle periods (also referred to as white spaces ${ }^{1}$ on the channel and opportunistically transmit its frames. However, it must ensure that the probability of its interference with any forthcoming PU transmission is below a predefined threshold. Many of the opportunistic channel access scheme for SUs proposed in the literature uses channel idle time distribution to decide when and how long to transmit when a white space is detected. However these schemes assume that either the start of idle period is known to the $\mathrm{SU}$, or the secondary network transmission is slotted and the SU senses the channel at the beginning of each slot. This requires the SU to either continuously or repeatedly sense the channel even if it does not have any frames to transmit. This approach is therefore energy-inefficient, specially for small, battery operated devices. In [11, we had

\footnotetext{
${ }^{1}$ In this paper, we use the terms channel idle time and white space interchangeably.

Copyright is held by the author/owner(s).

CogART 2011, October 26-October 29, 2011, Barcelona, Catalonia, Spain. ACM X-XXXXX-XX-X/XX/XX.
}

proposed an opportunistic channel access scheme in which an SU senses the channel only when it has one or more frames to transmit. If the channel is sensed idle, it uses the residual idle time distribution to estimate its transmission duration such that the probability of interfering with the primary is below a predefined threshold. This scheme does not need to know when the sensed idle period started. We had derived the analytical formulations for (i) SU's average throughput per white space (ii) SU's average utilization per white space, and had validated the analytical results using simulations. We had presented the results when the scheme was used with synthetic traffic with low variability white space distributions (for which Squared Coefficient of Variation $\left(\mathrm{CoV}^{2}\right)$ is less than 1.0) such as 2-Erlang and Uniform distribution. However, from our simulation experiments, we found that the channel occupancy data (idle and busy time values) for real primary applications show high variability. In this paper, we assume that the SU has obtained raw data (of idle and busy duration) from PU network and present the methodology to be followed by the SU so that it can use our scheme based on residual white space distribution. We illustrate this methodology through an example Primary Network that runs VoIP and Web Browsing applications on a single shared channel in a simulation environment. We also derive the analytical formulation for the average throughput achieved by an SU and validate it using simulation.

\section{RELATED WORK}

The authors in 6] propose channel sensing and transmission strategies under the Partially-Observed Markov Decision Process (POMDP) framework and require synchronization between the Primary and Secondary transmission slot structures. In 9], the authors have used empirical PDF and CDF of a whitespace trace (idle period) (obtained using whitespace measurements in simulation) to compute the sensing duration and the number of frames that an SU should transmit on sensing a channel idle, subject to a maximum PU interference probability bound. The paper computes, both analytically and using simulations, the Effective Secondary Throughput, and Primary User Interference. In 13 also, the SU determines the optimal transmission duration on finding a channel idle subject to maximum interference probability threshold. Both [9] and [13] use the channel idle time distribution in their MAC protocol design and assume that the start of each idle period is known. The authors in 5. approximated a Semi-Markov model for WLAN channels 
with continuous-time Markov chain.

\section{OVERVIEW OF RESIDUAL IDLE TIME DISTRIBUTION}

In this section, we give a brief overview of the residual white space distribution-based scheme for SUs to opportunistically transmit on the primary channel. For details, please refer to 11 and 12 .

The channel occupancy due to PU activities is modeled as Alternating Renewal Process (ARP), in which the channel state alternates between busy and idle states. An ARP can be treated as a renewal process in which a renewal cycle consists of a busy and an idle period, and such cycle repeats itself over time. Let $B, I$ and $X$ represent the random variables denoting channel busy, idle and the cycle time respectively. Hence, $X=B+I$. Let $F_{B}, F_{I}$ and $F_{X}$ represent cumulative distribution of the channel busy, idle and cycle time respectively ${ }^{2}$. We use $E[$.$] to denote the mean value of$ a random variable. We assume that $\mathrm{SU}$ knows the idle and busy time distributions along with their parameter values. Let $f_{R I}$ represent the residual idle time density function, given that the $\mathrm{SU}$ has sensed the channel idle. This is given by [14, pp. 331]

$$
f_{R I}(y)=\frac{1-F_{I}(y)}{E[I]}
$$

Then the residual idle time distribution function is given by

$$
F_{R I}(y)=\int_{0}^{y} f_{R I}(z) \mathrm{d} z
$$

Note that $F_{R I}(y)$ denotes probability that residual idle time is less than $y$. Hence the duration $y$, for which the SU transmits in the remaining white space (given that it has sensed the channel idle) while satisfying the interference probability constraint $\eta^{3}$ is obtained by solving the following inequality.

$$
F_{R I}(y) \leq \eta
$$

The maximum value of $y$ (which we denote as $y_{\max }$ ), for which the above inequality is satisfied, is taken as the duration for which the $\mathrm{SU}$ transmits in the remaining white space, once it has sensed the channel to be idle.

\section{OUR METHODOLOGY}

\subsection{Primary Channel Occupancy Modeling Us- ing Distribution Fitting}

Figure 1 shows the flow chart, which describes the steps to be followed to model the Primary channel occupancy using distribution fitting. The symbol $\mathrm{CoV}^{2}$ used in the flowchart is the square of coefficient of variation. We would like to emphasize that the computation of $F_{R I}$ (see (1) and (2)) requires closed form expression of the CDF of the fitted idle time distribution. If the fitted distribution does not have a closed form distribution function in general (such as Pareto, Gamma, Normal, Log Normal, Beta, etc.), then such distributions must be approximated using Phase type (PH) distributions and the approximated $\mathrm{PH}$ distribution should be used to compute $F_{R I}$.

${ }^{2}$ Distribution of $\mathrm{X}$ can be derived using convolution of distributions of I and B.

${ }^{3} \eta$ represents the upper bound on the probability of interference of SU's transmission with PU transmission.

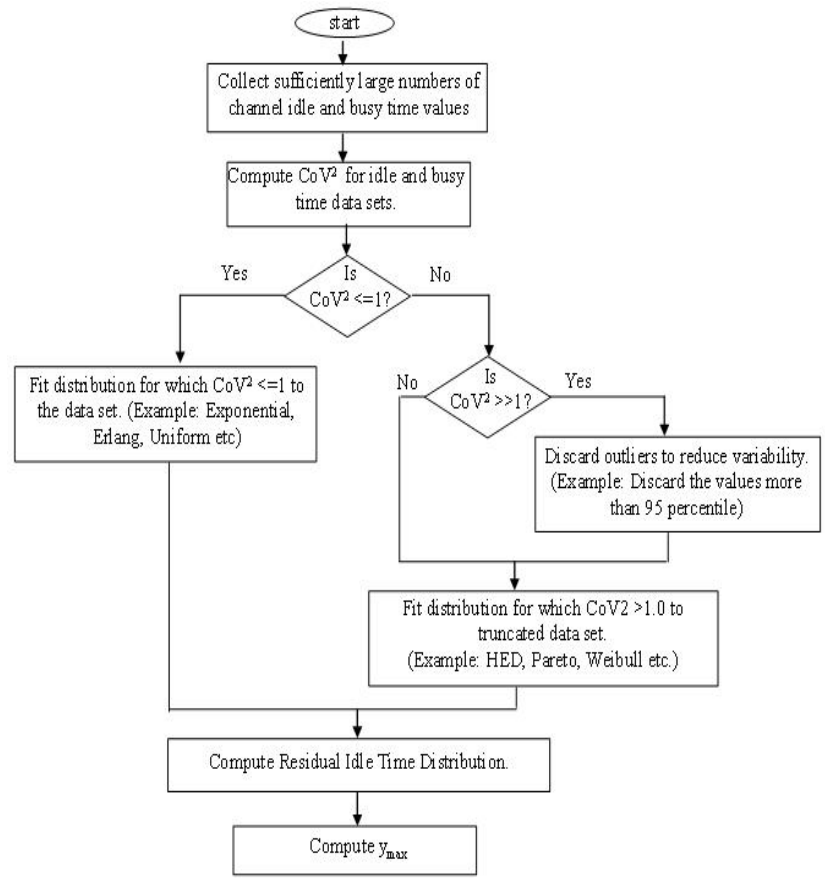

Figure 1: Methodology to Use Residual White Space Distribution-based Channel Access

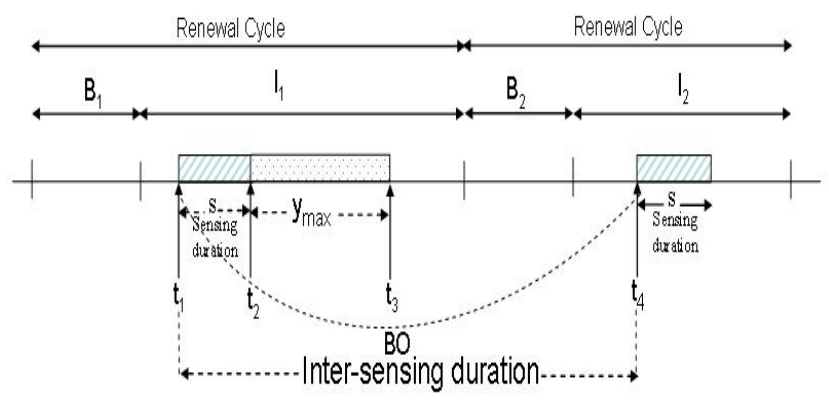

Figure 2: Opportunistic SU Transmission

\subsection{Opportunistic SU Transmission}

Whenever an SU's MAC layer receives one or more packets for transmission, it senses the channel for a duration of $s$. This sensing operation can be seen as random incidence on the channel (such as at time instants $t_{1}$ in Figure 24 If the channel is sensed idle (for the sensing duration $t_{1}$ to $t_{2}$ ), the node concludes the presence of white space which can be used for secondary frames transmission. It then transmits frames for $y_{\max }$ duration (from instant $t_{2}$ to $t_{3}$ ). There are three possible situations that can arise during this transmission process: (1) The channel is sensed busy during channel sensing (between $t_{1}$ and $t_{2}$ ) (2) The channel becomes busy (due to re-appearance of PU on the channel) during $y_{\max }$ transmission (between $t_{2}$ and $t_{3}$ ), and (3) SU successfully transmits for $y_{\max }$ duration (upto $t_{3}$ ) within the current white space. In all of these situations, the SU generates a random backoff value, which we denote by random variable

${ }^{4}$ The notion of SU "incidenting on the channel" refers to the action of SU sensing the channel to find whether it is idle or busy. 
$B O$. This value is generated using exponential distribution with mean $E[B O]$. The SU then computes the next sensing instant by adding the $B O$ value to the previous sensing time. For example, in Figure 2 , after transmitting successfully for $y_{\max }$ duration (upto $t_{3}$ ), the $\mathrm{SU}$ generates $B O$ value and computes the next sensing instant as $t_{4}=t_{1}$ (the previous sensing time) $+B O$. Since SU has already transmitted upto time $t_{3}$, it actually backs off for duration $\left(t_{4}-t_{3}\right)$ and senses the channel again at time instant $t_{4}$ so that the intersensing duration $\left(t_{4}-t_{1}\right)$ is exponentially distributed with mean $E[B O]^{5}$

It is important to ensure that the sensing process is Poisson because the residual idle time distribution is derived based on the assumption that the SU incidences on the channel (i.e. accesses the channel to sense it) randomly (see 14 , pp. 331]). One of the drawbacks of backing off is that the SU may miss some part of the white spaces during backoff. An alternative approach to backing off is to continuously sense the channel so as not to miss any white space. However, this approach is highly energy in-efficient (specially for battery operated small mobile SUs, and cognitive sensor networks) because the SU devices need to sense the channel even if they have no frame to transmit. On the contrary, the SU which uses the proposed residual idle time based channel access scheme senses the channel only when it has a frame to transmit, and consequently, have significantly lower sensing overhead per transmitted frame.

\section{ANALYTICAL FORMULATION OF PER- FORMANCE METRICS}

In this section, we present the analytical formulations for various performance metrics of a cognitive network based on our model.

Average SU Throughput per white space (ATHPWS) (in frames per white space), given that the SU has sensed the channel idle, is given as:

$$
A T H P W S=\frac{R *\left\{y_{\max }\left(1-F_{R I}\left(y_{\max }\right)\right)+\int_{q=0}^{y_{\max }} q f_{R I}(q) \mathrm{d} q\right\}}{S}
$$

Here, $y_{\max }$ is the maximum transmission duration within a white space as computed using (3), $R$ is the channel data rate (in bits per second), and $S$ is the sum of SU data and acknowledgment frames sizes (in bits). $f_{R I}$ is given by (1). The first term in the numerator captures the average duration for which the $\mathrm{SU}$ would transmit for the $y_{\max }$ duration and the second terms represents the average duration for which the SU transmits for less than $y_{\max }$ duration (because PU reappears before SU transmits for $y_{\max }$ duration). Note that $\left(1-F_{R I}\left(y_{\max }\right)\right)$ is the probability of successful transmission for $y_{\max }$ duration without interfering with PU. Please refer to 11 and 12 for more details.

In order to derive the analytical expression for average throughput of SU, we note that distribution of the renewal cycle (say $W$ ) in which the SU incidences (to sense the channel state) is different from the distribution of the other renewal cycles $X]^{6}$ The density of $W$ can be derived using

\footnotetext{
${ }^{5}$ Note that it is possible that $\left(t_{1}+B O\right)<t_{3}$, i.e., the generated $B O$ value is less than $\left(s+y_{\max }\right)$; in such a case, the $\mathrm{SU}$ generates a new value of $B O$ (say, $\left.B O_{1}\right)$, and computes the next sensing time value as $\left(t_{1}+B O+B O_{1}\right)$. These steps are repeated till the next sensing time value exceeds $t_{3}$.

${ }^{6} W$ is likely to be larger than $X$ as larger cycles are more
}

the density of $X$ (see 14, pp. 330, (6.22)]). We assume that the busy time values of the channel are constant (say $b$ ). From our observation of primary traffic (using simulation), we found that most of the time the primary sends a constant size packet (usually MTU size) and hence this assumption is quite practical. Hence, we represent $W=I_{W}+b$, where $I_{W}$ is the distribution of the idle period within the cycle $\mathrm{W}$. The density of $I_{W}$ (say $f_{I_{w}}$ ) can be derived using the density of $W$ (see 14 pp. 151, (3.55)]). Using the above densities, we can compute the long term probability $\left(P_{I_{W}}\right)$ that the SU's sensing falls in the idle period of the cycle $W$, which is given as:

$$
P_{I_{W}}=\frac{E\left[I_{W}\right]}{E[W]}
$$

As explained in Section 4.2 SU does not transmits if channel is idle for less than the sensing period $s$, i.e., if the channel becomes busy while the SU is sensing. Also, the SU backs off exponentially with mean backoff duration of $E[B O]$. But sometimes the backoff duration will be smaller than $\left(s+y_{\max }\right)$, in which case the SU does not transmit and continues to back off until it incidents beyond $\left(s+y_{\max }\right)$. Thus, we need to take the above two cases into consideration (when the SU would not transmit) while computing the average throughput.

Let us consider a sufficiently large duration $T$ over which we want to find the average throughput of SU. The number of incidences in this period would be $T / E[B O]$ (since the backoff is exponential with mean $E[B O])$. Out of these incidences, fraction of $P_{I_{W}}$ would be in the Idle period, and some of these incidences (corresponding to the two cases stated above) will result in no transmission. The average throughput of SU $(\gamma)$ (in frames per second) can be computed as follows:

$$
\begin{aligned}
\gamma & =\left[\frac{P\left\{B O>\left(s+y_{\max }\right)\right\} * P(R I>s) * P_{I_{W}} *\left(\frac{T}{E[B O]}\right)}{T}\right] \times \\
& =\left[\frac{P\left\{B O>\left(s+y_{\max }\right)\right\} * P(R I>s) * P_{I_{W}}}{A T H P W S}\right] \times A T H P W S
\end{aligned}
$$

Note that in the first expression, the numerator of the term in square bracket represents the average number of incidences of $\mathrm{SU}$ in white spaces in which it gets to transmit in a long duration $T$. In our work, we have taken the back off parameter value $(E[B O])$ to be equal to mean renewal cycle time $E[X]$ (which is equal to $E[B]+E[I]$ ).

\section{SIMULATION EXPERIMENTS}

\subsection{Simulation Model}

We have used OPNET simulator 2 for simulation experiments. The simulation model consist of a single channel of $4 \mathrm{Mbps}$ data rate, which is shared by two pairs of PUs. One pair of PU nodes run VoIP application (over UDP and $\mathrm{RTP}$ ) in peer-to-peer mode, whereas the second pair of PU nodes run a web browsing application using HTTP 1.1 protocol (over TCP/IP) in client-server mode. Therefore, the channel carries a mix of VoIP and HTTP traffic. The VoIP parameters are the commonly used default OPNET parameters, whereas the web application parameters used in simulations are from 15]. For VoIP application, the encoding scheme is G.723.1 with 5.3Kbps bit rate, the incoming and

$\overline{\text { likely to be intercepted by SU }}$ 14, pp. 329] 
Table 1: Summary Statistics of White Spaces for (VoIP+HTTP) Application

\begin{tabular}{|c|c|c|c|c|c|c|c|c|}
\hline Parameters & Minimum & Maximum & Mean & Median & Variance & 95 Percentile & CoV $^{2}$ & Skewness \\
\hline Values & $0.168 \mathrm{~ms}$ & $45.880 \mathrm{~ms}$ & $4.143 \mathrm{~ms}$ & $1.88 \mathrm{~ms}$ & $0.031357 \mathrm{~ms}$ & $19.88 \mathrm{~ms}$ & 1.826631 & 3.096771 \\
\hline
\end{tabular}

outgoing silence period durations (in seconds) are exponentially distributed with parameter 0.65 seconds, the incoming and outgoing talk spurt lengths (in seconds) are exponentially distributed with parameter 0.352 seconds, and one voice frame is transmitted per packet. For Web browsing application using HTTP 1.1 protocol, the page inter arrival time (in seconds) is Weibull distributed (scale $=0.67$, shape $=0.53$ ), the number of objects per page is Pareto distributed (location $=0.38$, shape $=0.85$ ), and size of each object is exponentially distributed with mean 1272 bytes. The PU nodes use slotted TDMA protocol to coordinate access to the shared channel. In addition to the Primary Users, the model has one pair of Secondary Users (SUs) which uses the proposed residual white space distribution based scheme to opportunistically transmit on the channel, while restricting its probability of interference to PU transmission below a predefined threshold $(\eta)$.

We have simulated saturated SU traffic, i.e., the SU always have enough frames to consume the available white space. SU frame size is 1024 bits and acknowledgment frames size is 128 bits. The simulations are first run without enabling the secondary transmissions to obtain the channel occupancy data (idle and busy periods) due to PU transmissions. Then, the obtained data is statistically analyzed and an appropriate white space distribution is fitted to the channel idle time values using the guidelines specified in Figure 1. This white space distribution is used by SUs to compute the residual white space distribution. Once the residual white space distribution is known, the simulations are run again where-in SUs opportunistically transmit on the channel using the residual white space distribution based channel access scheme.

\subsection{White Space Characterization}

The idle time (white space) data obtained using simulation shows high variability with Squared Coefficient of Variation $\left(\mathrm{CoV}^{2}\right)$ equal to 3.315 . In our experiments, we have taken 95 percentile of idle time data so as to remove the outliers and reduce the variability of the data. The $\mathrm{CoV}^{2}$ of the truncated data set is 1.826 . Table 1 shows the statistic summary of 95 percentile of idle time data values. A large number of idle values are quite small (values upto 90 percentile are less than $9.88 \mathrm{~ms}$ ) but a reasonable number of idle values are large (the last 10 percentile values lie between $9.88 \mathrm{~ms}$ and $45.88 \mathrm{~ms}$ ). The fact that the median of the data set is considerably smaller than the mean value and coefficient of skewness is greater than 1.0, indicates that even the truncated data is right-skewed, although with lesser variability.

One of the widely accepted approaches to model the characteristics of highly variable data (with $\mathrm{CoV}^{2}>1.0$ ) is to use Hyper Exponential Distribution (HED) [4]. An $r$-phase HED is a mixture of $r$ exponentials, each with associated rate and probability parameters. HED has been used to model the silence durations (white spaces) in voice communications $(3,8])$, as well as web traffic $(\sqrt{7})$. We have fitted a 2-phase HED to the measured idle time data set
Table 2: Comparison of Statistics of the Idle Data and the Fitted 2-HED

\begin{tabular}{|c|c|c|c|}
\hline & $\mathbf{E}[I]$ & $\mathbf{E}\left[I^{2}\right]$ & $\mathrm{CoV}^{2}$ \\
\hline Data & $4.143 m s$ & $48.5 \mu s$ & 1.826 \\
\hline 2-HED & $4.134 m s$ & $57.1 \mu s$ & 2.329 \\
\hline RE (\%) & 0.21 & 17.77 & 27.5 \\
\hline
\end{tabular}

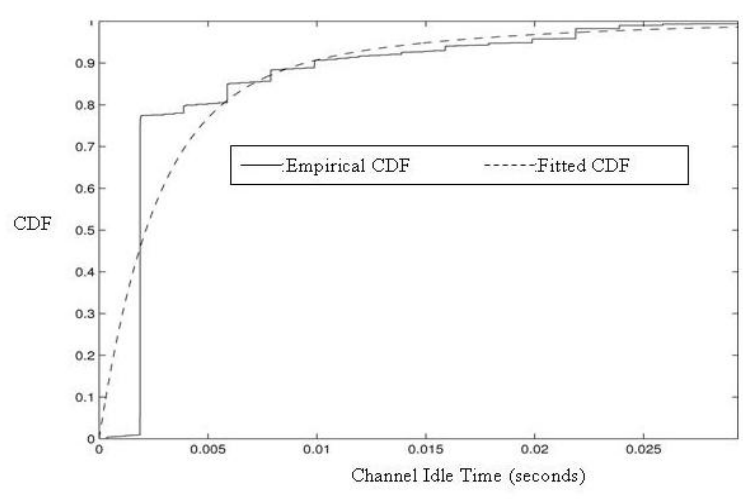

Figure 3: Comparison of Empirical CDF of Idle Time Data Set (Solid Curve) and the Fitted (2-HED) Distribution (Dashed Curve)

using a tool called EMpht 1, which is widely used to fit phase type distributions. The fitted parameter values are: $\left(\beta_{1}=0.808089, \lambda_{1}=400.45 \mathrm{sec}^{-1}\right)$ for first exponential, and $\left(\beta_{2}=0.191911, \lambda_{2}=90.3 \mathrm{sec}^{-1}\right)$ for second exponential, where $\lambda$ and $\beta$ denotes the rate and probability values of the exponentials. The data set size was 23173 values and the log likelihood of the 2-HED fit was 105370.08 . The large data set precludes us from using quantitative Goodness of Fit (GoF) tests such as Chi-square test and KS test. Therefore, as suggested in [10], we compare the first order statistic and CDF of the fitted 2-phase HED with that of the idle time data set to assess the GoF. Table 2 shows the comparison of the first order statistics. The last row of the table shows the percentage of Relative Error (RE) 7] between the statistic values of the measured data set and the fitted 2-HED. We note that the first moment matches reasonably well whereas the second moment differs by $17.77 \%$. Authors in 7] have also reported similar deviations for highly variable HED fits. Figure 3 shows the CDF of the fitted distribution and the idle data set.

For the busy time data set, the total number of values obtained were 24762 , out of which $94.4 \%$ of the values were constant $(120 \mu \mathrm{s}), 4 \%$ of the values were less than $120 \mu \mathrm{s}$ and $1.5 \%$ of the values were greter than $120 \mu \mathrm{s}$. Therefore, we take the busy periods as constant $b(b=120 \mu s)$ and do not fit any distribution to the busy time data set. We are not providing the graph for the empirical CDF of busy time 

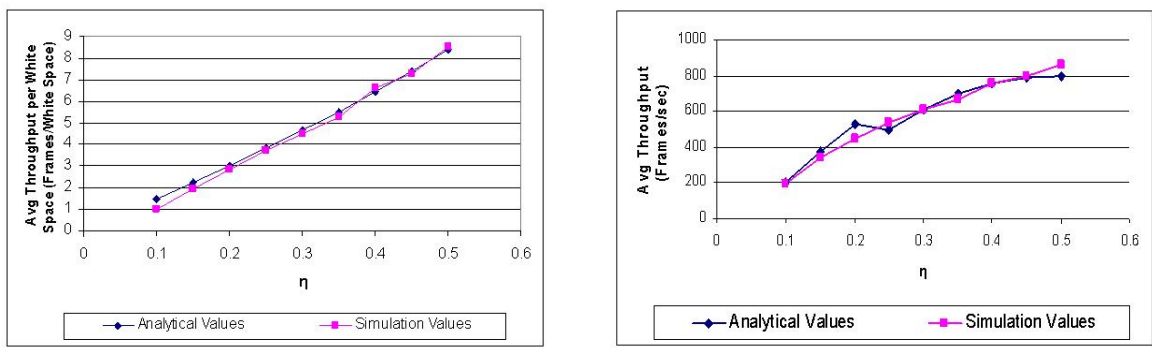

Figure 4: Avg SU Throughput per White Space

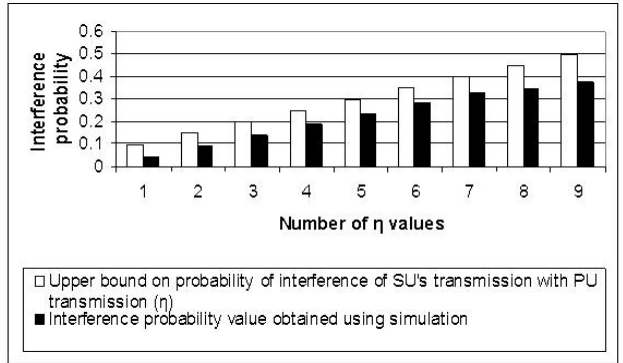

Figure 7: Probability of Interference of SU's Transmissions with PU

values for lack of space.

Since we have fitted 2-HED to the idle time values, we next specify the analytical formulations for the performance metrics when 2-HED is used as white space distribution.

\subsection{Analytical Formulations for 2-Phase HED Channel Idle Time}

For 2-phase HED fitted to idle time data values (with parameters $\left.\beta_{1}, \lambda_{1} ; \beta_{2}, \lambda_{2}\right)$, the residual idle time is also 2 HED distributed with same rate parameter values $\left(\lambda_{1}\right.$ and $\lambda_{2}$ ) but different probability values $\left(\alpha_{1}\right.$ and $\left.\alpha_{2}\right)$, which are given below. The density and the distribution of residual idle time can be derived in similar manner as described in 12 for other distributions, and are given as follows:

$$
f_{R I}(y)=\sum_{p=1}^{2} \alpha_{p} \lambda_{p} e^{-\lambda_{p} y}
$$

and

$$
F_{R I}(y)=\sum_{p=1}^{2} \alpha_{p}\left(1-e^{-\lambda_{p} y_{\max }}\right)
$$

Here,

$$
\alpha_{r}=\frac{\left(\beta_{r} / \lambda_{r}\right)}{\sum_{p=1}^{2}\left(\beta_{p} / \lambda_{p}\right)} \quad r=1,2
$$

and

$$
\alpha_{1}+\alpha_{2}=1
$$

Using equations (3) and (8), we obtain $y_{\max }$ by solving the following inequality for the maximum value of $y$ that satisfies the PU interference constraint:

$$
\alpha_{1} e^{-\lambda_{1} y}+\alpha_{2} e^{-\lambda_{2} y}+(\eta-1) \geq 0
$$

For the obtained value of $y_{\max }$, the expression for ATHPWS can be written as follows:

$$
A T H P W S=\left(\frac{R}{S}\right)\left[\frac{\alpha_{1}\left(1-e^{-\lambda_{1} y_{\max }}\right)}{\lambda_{1}}+\frac{\alpha_{2}\left(1-e^{-\lambda_{2} y_{\max }}\right)}{\lambda_{2}}\right]
$$

To derive expression for average throughput $(\gamma)$ of SU, we can first derive that:

$$
\begin{gathered}
E[X]=\frac{\beta_{1} e^{\lambda_{1} b}}{\lambda_{1}}+\frac{\beta_{2} e^{\lambda_{2} b}}{\lambda_{2}} \\
P_{I_{W}}=\frac{\left(\beta_{1} b\right) / \lambda_{1}+\left(2 \beta_{1}\right) / \lambda_{1}^{2}+\left(\beta_{2} b\right) / \lambda_{2}+\left(2 \beta_{2}\right) / \lambda_{2}^{2}}{\left(2 \beta_{1} e^{\lambda_{1} b}\right) / \lambda_{1}^{2}+\left(2 \beta_{2} e^{\lambda_{2} b}\right) / \lambda_{2}^{2}} \\
P(R I>s)=\alpha_{1} e^{-\lambda_{1} s}+\alpha_{2} e^{-\lambda_{2} s} \\
P\left\{\left(B O>\left(s+y_{\max }\right)\right\}=e^{\frac{-\left(s+y_{\max }\right)}{E[X]}}\right.
\end{gathered}
$$

We can then derive the expression for average $\mathrm{SU}$ throughput $(\gamma)$ using 6 .

\subsection{Simulation Results}

Figure 4 and Figure 5 compare the analytically estimated and simulation-based performance parameters for different PU interference probability constraints $(\eta)$. We note from the figures that the simulation values match reasonably well with the analytical values (except for $\eta=0.2$ in Figure 5). We observe that the larger the value of $\eta$ (i.e. larger acceptable PU interference), the higher the average SU throughput per white space, and average throughput of the SU. This is so because the analytical formulation (11) predicts larger transmission times (i.e larger $y_{\max }$ values) for larger $\eta$, and therefore, SU transmits aggressively in each white space. However, such aggressive transmission by the SU leads to higher PU interference. For smaller values of $\eta$, the estimated values of $y_{\max }$ are smaller. Therefore the SU transmissions are conservative (so that it can meet the low interference requirement of the Primary Users), which leads to low white space utilization by the SU but low interference to the PU. Figure 6 shows the channel efficiency 7 when only $\mathrm{PU}$ uses the channel (shown in the graph as $\eta=0$ ) and when SU also opportunistically transmit on the channel for various $\eta$ values. We note that the total channel efficiency increases (as compared to when PU alone uses the channel) when SU also starts using the channel oppotrtunistically. However the increase is not very high for two reasons. First, the SU does miss some white spaces because of sochastic nature of the proposed scheme. In order to avoid continuous channel sensing, the scheme works with limited information (it has no knowledge of the time that has elapsed in the current white space), which has an impact on utilization of the channel white space. Second, since the scheme is designed to ensure that the interference probability constraint $(\eta)$ is not violated, the transmission duration $\left(y_{\max }\right)$ for $\mathrm{SU}$

${ }^{7}$ We define channel efficiency, or channel bandwidth utilization, as the ratio of the achieved channel throughput and the maximum channel throughput (expressed in \%). 


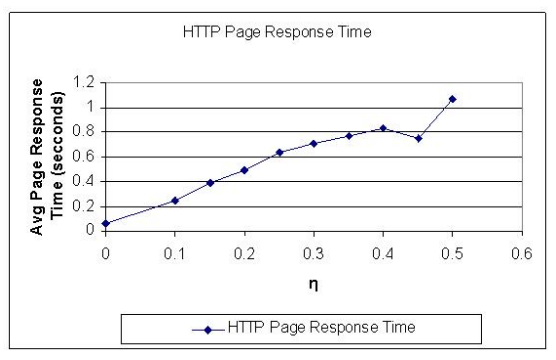

Figure 8: Average Page Response Time for Web Browsing Application (Sec)

is conservative, specially for low values of $\eta$. Figure 7 shows the interference probability of SU's transmissions obtained using simulation. We note that the SU does not violate the interference probability constraint for any $\eta$ value. Figure 8 shows the average HTTP page response time when only PU uses the channel $(\eta=0)$ and when the SU also starts using the channel opportunistically $(\eta=0.1$ to 0.5$)$. We note that as SU starts using the channel, the HTTP page response time increases. This is due to the fact that as SU transmits more aggressively (as $\eta$ value increases), the number of collisions with HTTP packets increases, and that leads to more retransmissions and increased retransmit time out value at HTTP client node (PU).

In general, as SU starts using the primary channel opportunistically and as $\eta$ increases, the PU application performance will deteriorate. However, in Figure 9, we note that as the SU starts using the primary channel opportunistically (at $\eta=0.1$ ), the end-to-end VoIP packet delay marginally decreases initially by approximately $3.5 \mathrm{~ms}$ (as compared to scenario when only PU uses the channel $(\eta=0))$. This is due to the following reason. Whenever an SU packet collides with the HTTP packet, the SU node backs off and the HTTP client goes into congestion control mode. TCP layer at the HTTP client doubles its RTO value and decreases its congestion window size, due to which the HTTP client offers less load on the channel. Therefore, the VoIP application gets more bandwidth until either the SU node tries to re-access the channel after its backoff or TCP congestion window size of HTTP client increases. As $\eta$ increases beyond 0.15 , the $\mathrm{SU}$ transmission becomes more aggressive and start impacting the VoIP application as well. Therefore, we notice the small increase in packet end-to-end delay for $\eta>0.15$. Figure 10 shows the packet delay variation for VoIP application. We note from the figure that delay variation has similar property as packet delay.

\section{CONCLUSIONS AND FUTURE WORK}

We have presented a methodology to be used to fit idle and busy time distributions to channel occupancy data and to use the residual white space distribution-based opportunistic channel access scheme in realistic Primary Networks. We have shown that using the described scheme, the channel efficiency increases due to SU transmissions, without violating the PU interference probability constraint. We have also shown that SU's opportunistic transmissions have minimal impact on PU applications, especially for low interference probability constraint. We are currently working on formulating the analytical expression for the average throughput

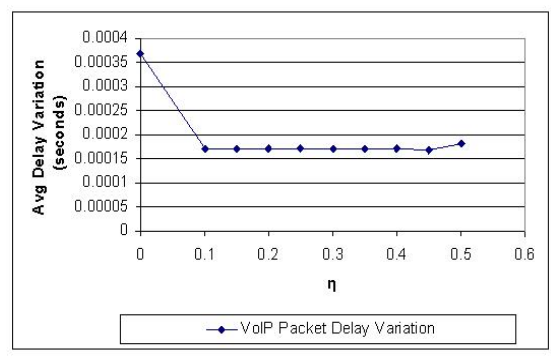

Figure 10: Average End to End Packet Delay Variation for VoIP Application (Sec)

of SU when the channel busy time values are not constants but have some specific distribution.

\section{REFERENCES}

\section{[1] The EMpht-programme.} http://home.imf.au.dk/asmus/pspapers.html.

[2] Opnet network simulator. http://www.opnet.com.

[3] F. Barcelo. Statistical Properties of Silence Gap in Public Mobile Telephony Channels with Application to Data Transmission. In Proceedings of IEEE ICC, 2001.

[4] A. Feldmann and W. Whitt. Fitting mixtures of exponentials to long tail distributions to analyze network performance models. Performance Evaluation (Elsevier), 31, 1998.

[5] S. Geirhofer, L. Tong, and B. M. Sadler. Cognitive Medium Access: Constraining Interference Based on Experimental Models. IEEE Journal on Selected Areas in Communications, 26(1), January 2008.

[6] A. T. Hoang, Y. C. Ling, D. Yong, Y. Zeng, and R. Zhang. Opportunistic Spectrum Access for Energy-Constrained Cognitive Radios. IEEE Transactions on Wireless Communications, 8(3), March 2009.

[7] R. E. A. Khayari, R. Sadre, and B. R. Haverkort. Fitting world-wide web request traces wth the EM-algorithm. Performance Evaluation (Elsevier), 52, 2003.

[8] P. OReilly and S. Ghani. Data Performance in Burst Switching When the Voice Silence Periods Have a Hyperexponential Distribution. IEEE Transactions on Communications, COM-35(10), October 1987.

[9] A. Plummer, M. Taghizadeh, and S. Biswas. Measurement Based Capacity Scavenging via Whitespace Modeling in Wireless Networks. In Proc. of IEEE GLOBECOM, 2009.

[10] A. Riska, V. Diev, and E. Smirni. Efficient fitting of longtailed data sets into hyperexponential distributions. In Proceedings of IEEE GLOBECOM, 2007.

[11] M. Sharma and A. Sahoo. Opportunistic Channel Access Scheme for Cognitive Radio System Based on Residual White Space Distribution. In Proc. of IEEE PIMRC, 2010.

[12] M. Sharma and A. Sahoo. Residual White Space Distribution Based Opportunistic Channel Access Scheme for Cognitive Radio Systems. Technical report, Indian Institute of Technology (IIT) Bombay, March 2010. http://www.cse.iitb.ac. in/internal/techreports/reports/TR-CSE-2010-30.pdf

[13] K. W. Sung, S. L. Kim, and J. Zander. Temporal Spectrum Sharing Based on Primary User Activity Prediction. IEEE Transactions on Wireless Communications, 9(12), December 2010 .

[14] K. S. Trivedi. Probability and Statistics with Reliability, Queuing and Computer Science Applications. John Wiley and Sons, Inc., 2nd edition, 2002.

[15] C. Zhu, Y. Wang, Y. Zhang, and W. Wu. Different Behavioral Characteristics of Web Traffic between Wireless and Wire IP Network. In Proceedings of IEEE ICCT, 2003. 